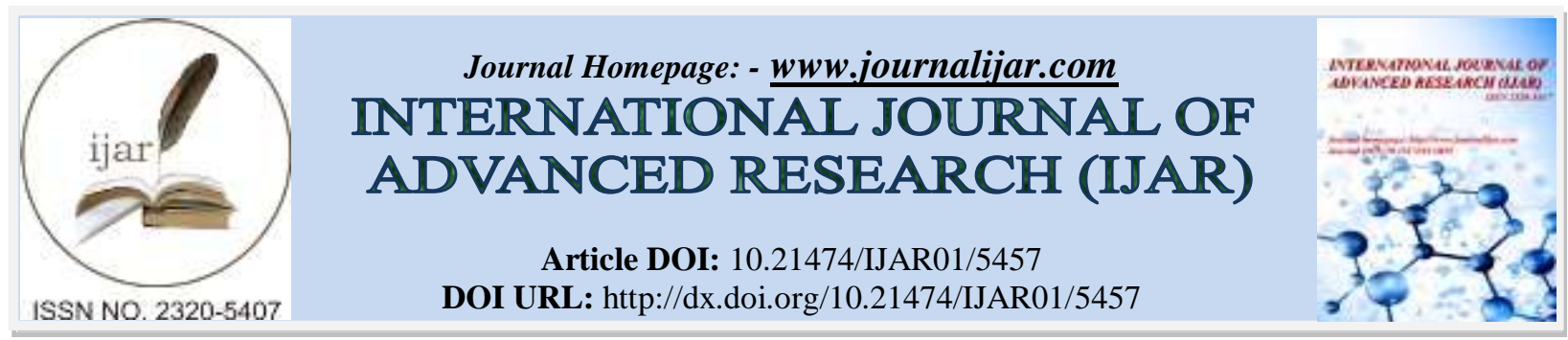

A SYSTEMATIC REVIEW

\title{
EFFECTIVENESS OF 2\% CHLORHEXIDINE ON REMOVAL OF E.FAECALIS BIOFILM IN ROOT CANAL: A SYSTEMATIC REVIEW.
}

\author{
Dr. Mubssira Shaikh, Dr. Pradeep Shetty, Dr. Monika Chawla and Dr. Ekta Senga, Dr. Sharon Coelho and \\ Dr. Neha Neekhara. \\ Department of Conservative Dentistry and Endodontics, Dr. D. Y. Patil Vidyapeeth (DPU), DR. D. Y. Patil Dental \\ College and Hospital, Pimpri, Pune-18
}

\section{Manuscript Info}

Manuscript History

Received: 17 July 2017

Final Accepted: 19 August 2017

Published: September 2017

\section{Abstract}

Copy Right, IJAR, 2017,. All rights reserved.

\section{Introduction:-}

Microorganisms play an important role in the etiology of pulpal and periapical lesions and various studies have clearly demonstrated it. ${ }^{1}$ The main goal of root canal treatment is the complete eradication of microorganisms and their by-products from the root canal system, though not always achieved. ${ }^{1}$ After root canal treatment, if Secondary infection occurs, it is caused by microorganisms and their by-products that resisted intracanal antimicrobial procedures and periods of deprivation, in treated canals. ${ }^{2,3}$ Hence, the use of an effective irrigant is necessary for effective removal of microorganisms from areas that are not accessible to instruments. ${ }^{4}$

Numbers of root canal irrigants have been developed in an attempt to reduce or eliminate the bacteria from the root canal system. The root canal irrigant at the very least should have antibacterial activity and a capacity to dissolve tissues. ${ }^{4}$ Such solutions should also be compatible with periradicular tissues, and capable of maintaining their therapeutic effect for a long period of time.

Sodium hypochlorite is the most commonly used irrigant, its tissue-dissolving and antibacterial properties have been well proven. However, it is not substantive and is highly irritating to periradicular tissues at higher concentrations. ${ }^{5}$ In addition; factors such as concentration ${ }^{6}$, temperature ${ }^{7}$ and $\mathrm{pH}^{8}$ greatly affect its efficacy.

Chlorhexidine has recently been introduced as an alternative irrigating solution. Chlorhexidine (CHX) is a broad spectrum antimicrobial agent active against aerobic and anaerobic bacteria. ${ }^{9}$ Chlorhexidine is a cationic molecule, which can be used during treatment.Furthermore, because of its cationic structure, chlorhexidine has a unique property named substantivity. CHX has strong binding affinity to the hydroxyapatite in dentin, enamel and cementum, and can be slowly released. ${ }^{10}$ This property gives CHX more longer-lasting bacteriostatic activity and the antibacterial efficacy of CHX has been shown to continue for up to $72 \mathrm{~h}$ after instrumentation. ${ }^{11}$ On the other hand, CHX lacks the ability to dissolve organic matter, which negatively affects its cleaning capacity.

Enterococcus faecalis (E. faecalis), a persistent organism that can survive as a monoculture in root canals. ${ }^{12}$ Its high prevalence in cases with post-treatment disease associated with virulence factors (aggregation substance, 
enterococcal surface proteins (Esp), gelatinase, cytolysin toxin, extracellular superoxide production, capsular polysaccharides, antibiotic resistance determinant) can facilitate the adherence of host cells and extracellular matrix, tissue invasions, immunomodulation effect and cause toxin mediated damage. ${ }^{12}$

Actual thinking has recommended the implementation of evidence-based dentistry, which organizes studies involving the systematic review or meta-analysis. Systematic reviews use a strict methodological approach to search, select, evaluate, and analyze original data from primary sources. ${ }^{13}$ Good scientific evidence is mandatory to elaborate clinical decisions, yet few systematic reviews or metaanalysis have been developed in Endodontics. ${ }^{14}$

Previous studies using in vitro experimental models have confirmed the antimicrobial efficacy of CHX against E. faecali, while others using different study designs have not found same results. ${ }^{14}$ Relevant clinical questions based on evidence regarding the resistance of $\mathrm{E}$. faecalis to $\mathrm{CHX}$ should be further discussed.

Therefore, the aim of this study was to review findings on the antibacterial efficacy of CHX on removal of E. Faecalis biofilm in root canal using systematic review.

\section{Focused Question:-}

What is the efficacy of $2 \%$ chlorhexidine for the removal of E.faecalis biofilm in freshly extracted human teeth using in vitro studies?

\section{Objective:-}

To evaluate the efficacy of $2 \%$ chlorhexidine on removal of E.faecalis biofilm in root canal.

\section{Methods:-}

Eligibility Criteria:-

Inclusion Criteria:-

1. Articles in English or those having detailed summary in English were included

2. Studies published between $1^{\text {st }}$ January 2005 to $31^{\text {st }}$ December 2015 were included

3. Studies in which setting was laboratory based were included

4. In vitro studies were included

5. Studies in which human extracted teeth were used as a sample were included

6. Studies in which $2 \%$ CHX was used as intervention were included

7. Studies that provide information about removal of E.faecalis biofilm using CHX were included.

\section{Exclusion Criteria:-}

1. Review, case reports, abstracts, letters to editors, editorials were excluded.

2. In vivo studies were excluded.

\section{The PICOS guidelines that were selected are:-}

$\mathrm{P}$ where Participants were included and this comprised of freshly extracted human teeth. I as the Intervention where this was considered as use a of $2 \%$ chlorhexidine. $\mathrm{O}$ as the outcome where it was assessed as the removal of E.faecalis biofilm in root canal. S as the study designs were included in vitro studies. And hence the PICOS are mentioned below:

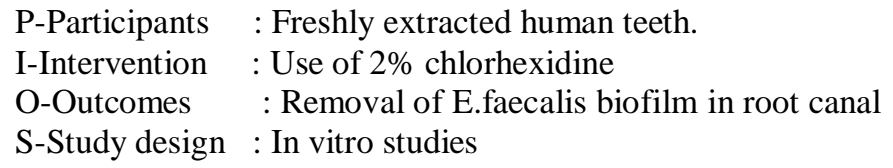

Information Sources:-

This review was designed using an analysis of comparative studies from a quantitative systematic review. Prospective studies were selected towards the efficacy of CHX against E. faecalis identified in endodontic infections. English-language articles were retrieved from electronic biomedical journal databases and handsearching records. The databases searched were PubMed and Google Scholar. 


\section{Search:-}

The following databases were searched on: PubMed (The limits used were all full text articles in English dated from $1^{\text {st }}$ January 2005 to December $31^{\text {st }} 2015$ ) and Google Scholar. For the electronic search strategy, the following terms were used as keywords in several combinations:

Keywords: Table no 1 \& Table no 2:-

Table No 1:- Table showing keywords used in this systematic review

\begin{tabular}{|l|l|}
\hline Biofilm /Biofilm removal & E.faecalis biofilm \\
\hline Chlorhexidine & $\begin{array}{l}\text { Antimicrobial agents, root canal irrigants, endodontic irrigant, , } \\
\text { disinfecting agent }\end{array}$ \\
\hline
\end{tabular}

Table No 2:-Table representing number of articles established using search strategy

\begin{tabular}{|l|l|l|l|l|}
\hline Sr. No. & Search strategy & \multicolumn{1}{|c|}{$\begin{array}{c}\text { Number of } \\
\text { articles }\end{array}$} & $\begin{array}{c}\text { Number of } \\
\text { selected } \\
\text { articles }\end{array}$ & \multicolumn{1}{|c|}{$\begin{array}{c}\text { After } \\
\text { Duplicate } \\
\text { Removal }\end{array}$} \\
\hline 1 & E.faecalis biofilm removal AND chlorhexidine & 3 & 2 & 2 \\
\hline 2 & $\begin{array}{l}\text { E.faecalis biofilm removal AND antimicrobial } \\
\text { agent OR chlorhexidine }\end{array}$ & 713 & 7 & 1 \\
\hline 3 & $\begin{array}{l}\text { E.faecalis biofilm removal AND chlorhexidine } \\
\text { OR root canal irrigants }\end{array}$ & 198 & 12 & 2 \\
\hline 4 & $\begin{array}{l}\text { E.faecalis biofilm removal AND endodontic } \\
\text { irrigant OR chlorhexidine }\end{array}$ & 712 & 5 & 0 \\
\hline 5 & $\begin{array}{l}\text { E.faecalis biofilm removal AND chlorhexidine } \\
\text { OR disinfecting agent. }\end{array}$ & 6 & 0 & 0 \\
\hline 6 & Other sources & 4 & 4 & 2 \\
\hline Total & & 1636 & 26 & 7 \\
\hline
\end{tabular}

\section{Study selection process:-}

In vitro and comparative studies were selected; however, only articles where the effect of $2 \%$ Chlorhexidine on removal of E. Faecalis biofilm in root canal in freshly extracted human teeth were included. Using different search strategies from the above mentioned key words and the combinations various electronic databases were searched. Total 1632 articles were identified through the database searching and 4 articles were identified through other sources. After thorough reading of tittles 1636 articles were selected. Further these records were assessed for any duplicates and 1608 articles were removed and 28 articles were screened for full text. Full text thorough readings of these, 21 articles were selected and were assessed for eligibility. Only 7 articles were qualified and 14 articles were excluded.8 articles were excluded as they use brain heart infusion (BHI) broth instead of freshly extracted human teeth and 2 articles were excluded as they used Trypticase soy agar plates were swabbed with E. Faecalis. 1 article was excluded as it was a review article. Another 3 articles were excluded as they did not qualify for the methodology selection.

\section{Data Collection Process:-}

Data collection process was done according to the consultation approved from our expert. First a Pilot Microsoft Excel Sheet was filled accordingly and then the expert was consulted for further progress. According to the data collected and the records selected the remaining Excel sheet was filled only with the data that was related to this study and retained.

\section{Data Items:-}

The headings under which the data was tabulated are Study ID where the number of studies that were selected was mentioned number wise. Various articles were included and so was the Name of the Author as an important factor. Year of Publication to mention and specify the fixed time interval that was selected. Study Design was mentioned as to specify the type of study design for example whether the study was in vivo, in vitro or in situ. Sample Size was mentioned to specify the number of participants included in the study. Population/Products were included and this comprised of freshly extracted human teeth. Intervention was selected as the use of $2 \%$ chlorhexidine. Time duration of irrigant was specified according to the various studies undertaken. Each group name and numbers were 
mentioned under Comparison heading according to various articles included. Result was mentioned according to the study's protocol and in the author's original words. Remark was expressed by the author of this systematic review.

The data items included were

1. Study ID -The number of studies that were selected were mentioned number wise

2. Author - The name of the author

3. Year of publication - The year in which the study was published

4. Study design - If the study was a control or a clinical trial, blinding

5. Sample size - No. of participants included in the study

6. Participant description - Single rooted extracted human teeth

7. Intervention Irrigants used in the trial

8. Time - Time of evaluation

9. Comparison - Green tea used in comparison with other controls

10. Outcome - Result of the study

11. Remarks - comments of the author

\section{Results:-}

Table 3:- Prisma Flow Chart

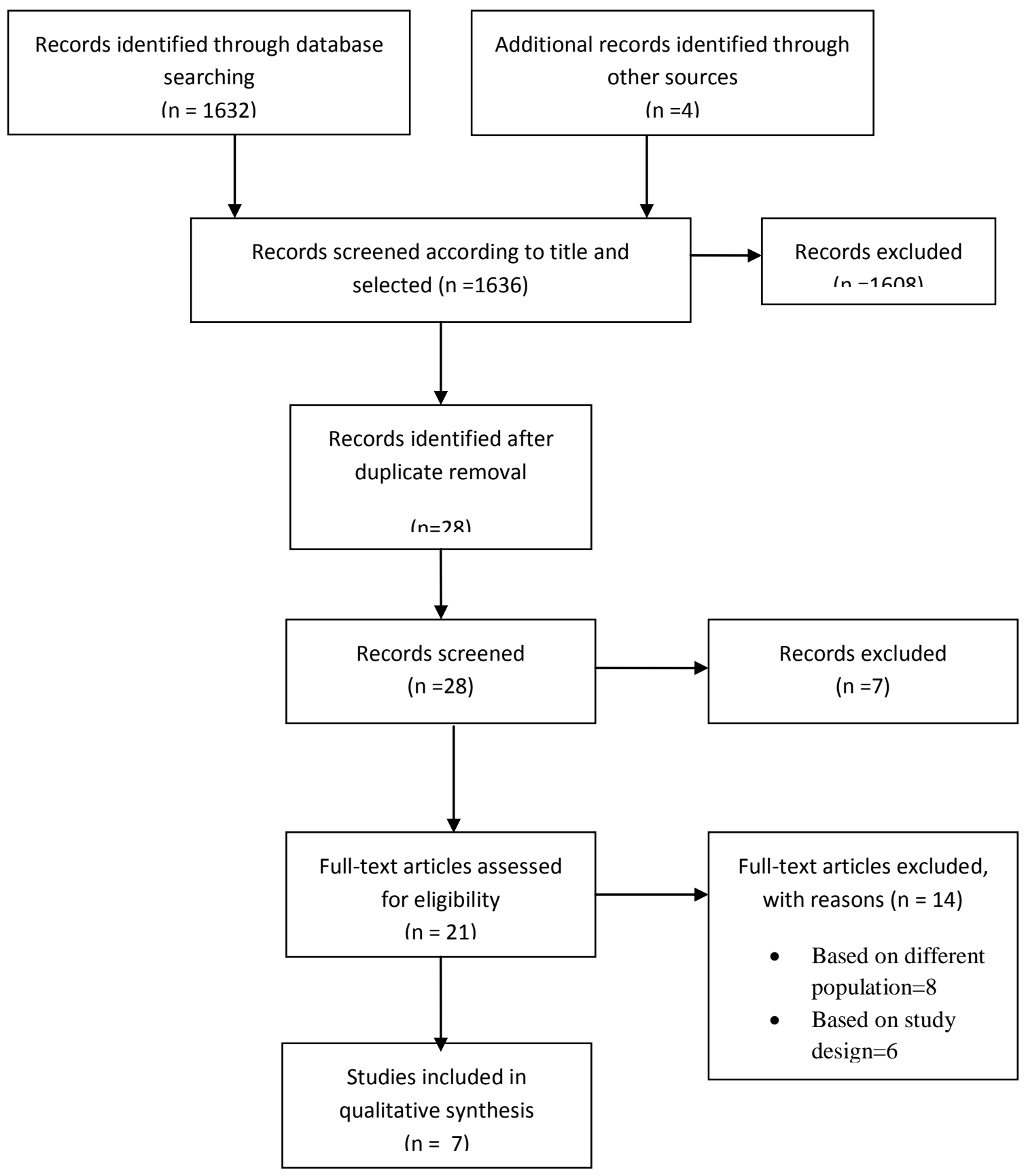




\section{Discussion:- \\ Summary of evidence:-}

The success of endodontic treatment is closely associated to the control of endodontic microbiota. Numbers of chemical irrigants have been suggested for use in the treatment of infected root canal. Several reviews may have been studied based on evidence regarding the resistance of $\mathrm{E}$. faecalis to CHX. This systematic review has been attempted to find the best available evidence relative to review findings on the antibacterial efficacy of CHX on removal of E. Faecalis biofilm in root canal system. However, it is difficult to draw conclusions from the articles selected as they cannot be compared directly due to the diversity of eligibility criteria's, assessment methods and outcomes.

Seven studies met the inclusion criteria established for the present investigation.

1. Mohammadi $\mathbf{Z}$ et al in 2008 compared the antimicrobial substantivity of BioPure MTAD, $2 \%$ chlorhexidine (CHX) and $2.6 \%$ sodium hypochlorite $(\mathrm{NaOCl})$. One hundred and ten dentin tubes prepared from human maxillary incisors were infected for 14 days with E.faecalis. The specimens were divided into five groups as follows: $\mathrm{CHX}$; BioPure MTAD; $\mathrm{NaOCl}$; infected dentin tubes (positive control); and sterile dentin tubes (negative control). Dentin chips were collected with round burs into Brain Heart Infusion (BHI) broth. After culturing, the number of colony-forming units (CFU) was counted. In all experimental groups, CFU was minimum after treatment (day 0), and the results obtained were significantly different from each other at any time period $(\mathrm{P}<0.05)$. After treatment, the NaOCI group and BioPure MTAD group showed the lowest and highest number of CFU, respectively. In conclusion, the substantivity of BioPure MTAD was significantly greater than $\mathrm{CHX}$ and $\mathrm{NaOCl}^{[15]}$.

2. Mehrvarzfar $\mathbf{P}$ et al in 2011 compared the effect of a diode laser and common disinfectants used in combination on mono-infected dental canals. One hundred and six single-rooted human premolars were prepared and contaminated with E.faecalis. After two weeks of incubation, samples were divided into two experimental groups $(n=48)$ and two control groups $(n=5)$. Absence of growth was seen only for MTAD plus laser treatment. Complete elimination of E. faecalis was seen only for the combination of MTAD with diode laser irradiation. Combination therapy with MTAD irrigation and diode laser irradiation, within the parameters used in this study, can be recommended as an effective treatment option for complete elimination of E. faecalis from the root canal system ${ }^{[16]}$.

3. Murad et al in 2012 reported that the antimicrobial efficacy of $2.5 \%$ and $5.25 \% \mathrm{NaOCl}, 2 \%$ gel and liquid CHX and MTAD against E.faecalis biofilms on human dentin. The antimicrobial activity of the test irrigants were assessed through CFU counts. Biofilm formation over the dentin surface was ensured by SEM analysis. Results showed no statistic difference among CHX gel, $2.5 \%$ and $5.25 \% \mathrm{NaOCl}$. However, the CHX liquid and MTAD were less effective than $2.5 \%$ and $5.25 \% \mathrm{NaOCl}$. Only CHX liquid and MTAD had differences in its efficacy depending on the time. The most effective irrigants in eliminating E. faecalis biofilms were $2.5 \%$ and $5.25 \% \mathrm{NaOCl}$ and $2 \% \mathrm{CHX}$ gel, at all the tested time intervals, in comparison to CHX liquid and MTAD ${ }^{[17]}$.

4. Hasheminia $\mathbf{S}$ et al in 2013 observed antibacterial activity of common root canal irrigants with a combination technique against intratubular E.faecalis. Seventy five specimens were contaminated with E. faecalis and divided into five experimental groups $(n=15)$. These groups were irrigated with $2 \%$ chlorhexidine (CHX), $3 \%$ hydrogen peroxide (H2O2), 5.25\% sodium hypochlorite $(\mathrm{NaOCl}), \mathrm{CHX} / \mathrm{H} 2 \mathrm{O} 2$ and sterile saline (control). Surface and deep dentinal chips were collected for each sample. After incubation, the numbers of colonyforming units (CFUs) were counted. In the surface dentin, $\mathrm{CHX}, \mathrm{NaOCl}$, and $\mathrm{CHX} / \mathrm{H} 2 \mathrm{O} 2$ had significantly higher antibacterial activity than $\mathrm{H} 2 \mathrm{O} 2(P<0.05)$. In the deep dentin, $\mathrm{NaOCl}$ and $\mathrm{CHX} / \mathrm{H} 2 \mathrm{O} 2$ had significantly higher antibacterial activity than $\mathrm{CHX}$ and $\mathrm{H} 2 \mathrm{O} 2(P<0.05)$. CHX/H2O2 had similar antibacterial effectiveness to $\mathrm{NaOCl}$ in both surface and deep dentinal tubules. This combination can be considered a potentially useful irrigant for root canal treatment ${ }^{[18]}$.

5. Valera MC et al in 2013 detected the antimicrobial activity of auxiliary chemical substances and natural extracts on $C$. albicans and E. faecalis inoculated in root canals. Seventy-two human tooth roots were contaminated with $C$. albicans and E. faecalis for 21 days. The groups were divided according to the auxiliary chemical substance into: G1) 2.5\% sodium hypochlorite ( $\mathrm{NaOCl}), \mathrm{G} 2$ ) $2 \%$ chlorhexidine gel (CHX), G3) castor oil, G4) glycolic Aloe Vera extract, G5) glycolic ginger extract and G6) sterile saline (control). The 
samples of the root canal were collected at different intervals: confirmation collection, at 21 days after contamination; 1st collection, after instrumentation; and 2nd collection, seven days after instrumentation. Microbiological samples were grown in culture medium and incubated at $37^{\circ} \mathrm{C}$ for 48 hours. $\mathrm{NaOCl}$ and $\mathrm{CHX}$ completely eliminated the microorganisms of the root canals. Reduction of $\mathrm{CFU} / \mathrm{mL}$ at the 1 st and $2 \mathrm{nd}$ collections for groups G1, G2, G3 and G4 was greater in comparison to groups G5 and G6. It was concluded that $2.5 \%$ sodium hypochlorite and $2 \%$ chlorhexidine gel were more effective in eliminating C. albicans and E. faecalis, followed by the castor oil and glycolic ginger extract. The Aloe vera extract showed no antimicrobial activity ${ }^{[19]}$.

6. María Ferrer-Luque $\mathbf{C}$ et al in 2014stated that effective final irrigation regimen is an important step in order to achieve better disinfection and ensure residual antimicrobial effects after root canal preparation. They compare the residual antimicrobial activity of $0.2 \%$ cetrimide, and $0.2 \%$ and $2 \%$ chlorhexidine in root canals infected with E.faecalis.After root canal preparation, root canals were irrigated with 17\%EDTA to remove the smear layer. The roots were randomly divided into three experimental groups (n526) according to the final

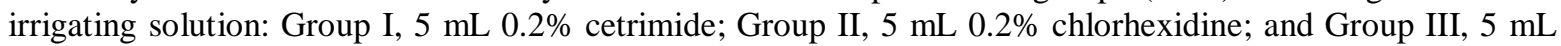
$2 \%$ chlorhexidine. Samples were collected for 50 days to denote the presence of bacterial growth. Differences among groups were tested using the log-rank test and the level of statistical significance was set at P,0.05. The highest survival value was found with $2 \%$ chlorhexidine, showing statistically significant differences from the other two groups. At 50 days, E. faecalis growth was detected in $69.23 \%$ specimens in Groups I and II, and in $34.61 \%$ specimens of Group III. There were no significant differences between $0.2 \%$ cetrimide and $0.2 \%$ chlorhexidine. Final irrigation with $2 \%$ chlorhexidine showed greater residual activity than $0.2 \%$ chlorhexidine and $0.2 \%$ cetrimide in root canals infected with E. faecalis ${ }^{[20]}$.

7. Noites $\mathbf{R}$ et al in 2014 testing various irrigants, sodium hypochlorite, chlorhexidine, and ozone gas, alone or in combination, were effective against E.faecalis and C.albicans. 220 single rooted teeth, were inoculated with C.albicans and E.faecalis.Sodium hypochlorite, chlorhexidine, and gaseous ozone alone were ineffective in completely eliminating the microorganisms. The association of chlorhexidine at $2 \%$ followed by ozone gas for 24 seconds promoted the complete elimination of Candida albicans and Enterococcus faecalis. Flow cytometry shows that ozone and chlorhexidine act differently, which could explain its synergic activity. This new disinfection protocol, combining irrigation with chlorhexidine at $2 \%$ and ozone gas for 24 seconds, may be advantageous when treating infected root canals ${ }^{[21]}$.

\section{Limitations:-}

These studies do not give concrete conclusions due to inadequate search of literature because of less access to search forums, failure to evaluate quality of studies, failure to exclude poorly designed studies, inappropriately combining heterogeneous studies, smaller sample sizes, availability of relevant articles in different languages other than English.

\section{Conclusion:-}

Within the present review limitations, It was shown that, $2 \% \mathrm{CHX}$ has a better efficacy when it is used in combination with ozone gas, this review also suggest that the antibacterial residual effect of $2 \%$ CHX would depend on its concentration and the length of its application time. It can be demonstrated that $2 \%$ CHX was a potential useful solution for the elimination of E. Faecalis biofilm due to its substantivity. Also, it has shown good efficacy when compared to other irrigants in decreasing the microbial load from the surface dentin of root canal system.

However two reviews shown that the substantivity of BioPure MTAD was significantly higher than CHX. Also the combination therapy consisting of irrigation and laser irradiation, Biopure MTAD, is an effective treatment option as compared to CHX for eliminating E. faecalis from the root canal system.

In summary, the disinfection of the root canal system produced by action of CHX reduces the remaining endodontic microbiota, which favors the achievement of a higher level of success of the endodontic treatment. 


\section{References:-}

1. X.-S. He and W.-Y. Shi, "Oral microbiology: past, present and future," International Journal of Oral Science, vol. 1, no. 2, pp.47-58, 2009.

2. P. N. R. Nair, "On the causes of persistent apical periodontitis: a review," International Endodontic Journal, vol. 39, no. 4, pp. 249-281, 2006.

3. 3: J. F. Siqueira Jr. and I. N. R^oc, as, "Clinical implications and microbiology of bacterial persistence after treatment procedures,"Journal of Endodontics, vol. 34, no. 11, pp. 1291-e3, 2008.

4. 4: Haapasalo M, Shen Y, Qian W, Gao Y (2010) Irrigation in endodontics. Dent Clin North Am 54, $291-312$.

5. 5: Spangberg L, Engström B, Langeland K (1973) Biologic effects of dental materials. 3. Toxicity and antimicrobial effect of endodontic antiseptics in vitro. Oral Surg Oral Med

6. Oral Pathol 36, 856-871.

7. 6: Berber VB, Gomes BP, Sena NT, Vianna ME, Ferraz CC, Zaia AA et al. (2006) Efficacy of various concentrations of $\mathrm{NaOCl}$ and instrumentation techniques in reducing Enterococcus faecalis within root canals and dentinal tubules. Int Endod J 39, 10-17.

8. 7: Sirtes G, Waltimo T, Schaetzle M, Zehnder M (2005) The effects of temperature on sodium hypochlorite short-term stability, pulp dissolution capacity, and antimicrobial efficacy. J Endod 31, 669-671.

9. 8: Mercade M, Duran-Sindreu F, Kuttler S, Roig M, Durany N (2009) Antimicrobial efficacy of $4.2 \%$ sodium

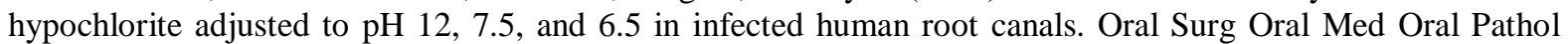
Oral Radiol Endod 107, 295-298.

10. 9: Jeansonne MJ, White RR (1994) A comparison of $2.0 \%$ chlorhexidine gluconate and $5.25 \%$ sodium hypochlorite as antimicrobial endodontic irrigants. J Endod 20, 276-278.

11. 10: Greenstein G, Berman C, Jaffin R (1986) Chlorhexidine. An adjunct to periodontal therapy. J Periodontol 57, 370-377.

12. 11: White RR, Hays GL, Janer LR (1997) Residual antimicrobial activity after canal irrigation with chlorhexidine. J Endod 23,229-231.

13. 12: Ro` $\mathrm{c}_{s}$ as IN, Siqueira JF Jr, Santos KR. Association of Enterococcus faecalis with different forms of periradicular diseases. J Endod 2004;30:315-20.

14. 13: Siwek J, Gourlay ML, Slawson DC, Shaughnessy AF. How to write an evidence-based clinical review article. Am Fam Physician. 2002;65:251-8.

15. 14: Law A, Messer H. An evidence-based analysis of the antibacterial efficacy of intracanal medicaments. J Endod. 2004;30:689-94.

16. 15: Mohammadi Z, Shahriari S. Residual antibacterial activity of chlorhexidine and MTAD in human root dentin in vitro. J Oral Sci. 2008 Mar;50(1):63-7.

17. 16: Mehrvarzfar P, Saghiri MA, Asatourian A, Fekrazad R, Karamifar K, Eslami G, Dadresanfar B. Additive effect of a diode laser on the antibacterial activity of $2.5 \% \mathrm{NaOCl}, 2 \% \mathrm{CHX}$ and MTAD against Enterococcus faecalis contaminating root canals: an in vitro study. J Oral Sci. 2011 Sep;53(3):355-60.

18. 17: Murad et al. Antimicrobial activity of sodium hypochlorite, chlorhexidine and MTAD® against Enterococcus faecalis biofilm on human dentin matrix in vitro. RSBO. 2012 Apr-Jun;9(2):143-50.

19. 18: Hasheminia S, Farhad AR, Saatchi M, Rajabzadeh M. Synergistic antibacterial activity of chlorhexidine and hydrogen peroxide against Enterococcus faecalis. J Oral Sci. 2013;55(4):275-80.

20. 19: Valera MC, Maekawa LE, de Oliveira LD, Jorge AO, Shygei É, Carvalho CA. In vitro antimicrobial activity of auxiliary chemical substances and natural extracts on Candida albicans and Enterococcus faecalis in root canals. J Appl Oral Sci. 2013 Mar-Apr;21(2):118-23.

21. 20: María Ferrer-Luque C, Teresa Arias-Moliz M, Ruíz-Linares M, Elena Martínez García M, Baca P. Residual activity of cetrimide and chlorhexidine on Enterococcus faecalis-infected root canals. Int J Oral Sci. 2014 Mar;6(1):46-9.

22. 21: Noites R, Pina-Vaz C, Rocha R, Carvalho MF, Gonçalves A, Pina-Vaz I. Synergistic antimicrobial action of chlorhexidine and ozone in endodontic treatment. Biomed Res Int. 2014; 2014:592423. 